\title{
Clinical relevance of glomerular lgM deposition in patients with lupus nephritis
}

\author{
Fengmei Wang ${ }^{1}$, Jirong Yu' ${ }^{1}$ Lei Zhang ${ }^{2}$, Yan Zhang ${ }^{2}$, Jie Zhang ${ }^{3}$, Bicheng Liu ${ }^{1 *}$ and Xiaowei Yang ${ }^{4,5^{*}}$
}

\begin{abstract}
Background: The aim of the study was to investigate the clinical relevance of IgM deposition in patients with lupus nephritis (LN) in a large cohort.

Results: 217 patients with renal biopsy-proven active LN were enrolled. The associations between glomerular lgM deposition and clinicopathological parameters were further analyzed. IgM deposition was positively correlated with glomerular C1q and C3 deposition moderately $(r=0.436, P<0.001 ; r=0.408, P<0.001$, respectively), and inversely correlated with plasma levels of C3 and CFH mildly $(r=-0.138, P=0.043 ; r=-0.147, P=0.037$, respectively). By multivariate analysis, we found that glomerular IgM deposition independently contributed to glomerular C3 deposition in patients with $\mathrm{LN}(\mathrm{OR}=2.002,95 \% \mathrm{Cl} 1.295-3.094, P=0.002)$. In addition, we also found that patients with IgM 0-2+ had similar plasma CFH levels, but in patients with IgM3+-4+, plasma CFH levels were significantly lower $(300.4 \pm 155.8 \mu \mathrm{g} / \mathrm{mL}$ vs. $429.9 \pm 187.5 \mu \mathrm{g} / \mathrm{mL}, P<0.001)$. Furthermore, patients with high density of glomerular lgM and low levels of CFH had heavier proteinuria, higher serum creatinine and lower plasma C3 levels $(5.7 \pm 3.1 \mathrm{~g} / \mathrm{d}$ vs. $4.7 \pm 3.5 \mathrm{~g} / \mathrm{d}, P=0.037 ; 150.1 \pm 121.0 \mu \mathrm{mol} / \mathrm{L}$ vs. $105.6 \pm 97.1 \mu \mathrm{mol} / \mathrm{L}, P=0.005 ; 0.3 \pm 0.2 \mu \mathrm{g} / \mathrm{L} \mathrm{vs} .0 .4 \pm 0.2 \mu \mathrm{g} / \mathrm{L}$, $P=0.04$, respectively), comparing with those with low density of glomerular lgM and low levels of CFH.
\end{abstract}

Conclusions: Our results suggested the involvement of glomerular deposited lgM in complement activation and renal injury in $L N$.

Keywords: IgM, Lupus nephritis, Complement factor H, Complement activation

\section{Background}

Lupus nephritis (LN) is the most common complication of systemic lupus erythematosus (SLE). The pathogenesis of LN involves initiation by immune complexes, activation of the immune system in the kidney, and the responses of renal parenchymal cells to these insults. Although LN is characterized by a "full-house" pattern of immune deposits, it is mostly suggested to be initiated by the glomerular deposition of nephritogenic IgG type autoantibodies at present [1-4].

\footnotetext{
*Correspondence: liubc64@163.com; yxw0537@163.com

${ }^{1}$ Institute of Nephrology, Zhong Da Hospital, Southeast University School of Medicine, Nanjing, China

${ }^{4}$ Department of Nephrology, Provincial Hospital Affiliated to Shandong

University, Jinan 250021, Shandong, China

Full list of author information is available at the end of the article
}

Glomerular IgM deposition occurs in a wide range of glomerular diseases. It was previously considered to be passively trapped in areas of glomerulosclerosis. However, recent studies found that IgM specifically bound insulted glomeruli and exacerbated renal injury. In mice deficient in the complement factor $\mathrm{H}(\mathrm{CFH})$, a model of non-sclerotic and nonimmune-complex glomerular disease, IgM was identified as binding to glomerular epitopes and contributing to the progression of glomerular damage [5]. In another animal model of adriamycininduced focal segmental glomerulosclerosis (FSGS), IgM deposition activated the complement system and mediated glomerular injury [6]. In the subsequent clinical studies, IgM deposition independently associated with worse renal outcomes in patients with various glomerular 
diseases, including FSGS, IgA nephropathy (IgAN), and diabetic nephropathy (DN) [7-9].

Since natural antibody IgM is suggested to bind to endogenous neoepitopes that are exposed after injury and exacerbates damage [10-13], whether it is involved in the pathogenesis of $\mathrm{LN}$ presented with various types of kidney injury deserves to be investigated. In our previous study, we found that plasma CFH levels in patients with $\mathrm{LN}$ at active phase were significantly lower than those in non-renal SLE patients or those in normal controls, and plasma CFH levels were negatively associated with SLEDAI scores and positively associated with serum C3 levels. In the remission phase of $\mathrm{LN}$, plasma CFH returned to the normal levels [14]. Whether the decreased CFH level in active LN leads to extra glomerular IgM deposition just as it did in $\mathrm{CFH}^{-1-}$ mice is also an interesting question.

In this study, we firstly investigated the clinical relevance of renal IgM deposition and the relationship of plasma CFH levels with glomerular IgM deposition in a large Chinese LN cohort.

\section{Results}

\section{Baseline data of patients with LN}

In total, 217 consecutive patients with renal biopsyproven active LN were enrolled. General clinical and renal histopathological profiles of the patients at renal biopsy are listed in Tables 1 and 2 . The age of the patients were $32.7 \pm 11.9$ years old. The female-to-male ratio was 5.2:1. At the time of renal biopsy, all the patients recieved oral predisone or intravenous methyprednisonlone. 23 (10.6\%) patients received mycophenolate mofetil combined with glucocorticoid, 12 (5.5\%) patients received tarcrolimus combined with glucocorticoid, the other 182 patients received no immunosuppressants.

According to the 2003 classification of LN, 7 patients were classified as Class II (3.2\%), 37 as Class III (17.1\%, including 12 as Class III +V), 126 as Class IV (58.1\%, including 7 as Class IV $+\mathrm{V})$, and 47 as Class V (21.7\%). None were Classes I and VI in this study.

\section{Glomerular IgM deposition and correlations with clinicopathological parameters in patients with LN}

Renal immunofluorescence profiles of the 217 patients at renal biopsy are listed in Table 3. Among the 217 patients, 33 (15.2\%) patients had no IgM deposition in glomeruli, whereas 184 patients had IgM deposition in glomeruli, including $72(33.2 \%)$ patients with IgM 1+, 81 (37.3\%) patients with IgM 2+, 29 (13.4\%) patients with IgM $3+$, and $2(0.9 \%)$ patients with IgM $4+$.

To explore the clinical implications of glomerular IgM deposition in LN, we analyzed the correlations between the intensity of IgM deposition and clinico-histological
Table 1 General clinical profiles of patients with lupus nephritis at renal biopsy

\begin{tabular}{|c|c|}
\hline Characteristic & Value \\
\hline Number of patients & 217 \\
\hline Age (mean \pm s.d.) (years) & $32.7 \pm 11.9$ \\
\hline Gender (female/male) & $182 / 35$ \\
\hline $\begin{array}{l}\text { The time between presentation of lupus nephritis and } \\
\text { biopsy (median, range) (months) }\end{array}$ & $23(0-214)$ \\
\hline Duration of follow-up (median, range) (months) & $49(6-240)$ \\
\hline SLEDAI (mean \pm s.d.) & $17.5 \pm 5.7$ \\
\hline Number of fever (non-infectious) (\%) & $66(30.4)$ \\
\hline Number of eruption (\%) & $120(55.3)$ \\
\hline Number of photosensitivity (\%) & $50(23)$ \\
\hline Number of oral ulcer (\%) & $64(29.5)$ \\
\hline Number of arthralgia (\%) & $118(54.4)$ \\
\hline Number of neurological disorder (\%) & $12(5.5)$ \\
\hline Number of anemia (\%) & $143(65.9)$ \\
\hline Number of thrombocytopenia (\%) & $66(30.4)$ \\
\hline Number of leukocytopenia (\%) & $101(46.5)$ \\
\hline Number of hematuria (\%) & $166(76.5)$ \\
\hline Number of leukocyturia (non-infection) (\%) & $113(52.1)$ \\
\hline Number of acute renal failure (\%) & $40(18.4)$ \\
\hline Hemoglobin (mean \pm s.d.) (g/L) & $101.9 \pm 25.8$ \\
\hline Urine protein (mean \pm s.d.) (g/24 h) & $4.3 \pm 3.2$ \\
\hline Serum creatinine (median, IQR) $(\mu \mathrm{mol} / \mathrm{L})$ & $84(68-129)$ \\
\hline C3 (mean \pm s.d.) $(\mathrm{g} / \mathrm{L})$ & $0.46 \pm 0.23$ \\
\hline C4 (mean \pm s.d.) $(\mathrm{g} / \mathrm{L})$ & $0.23 \pm 0.17$ \\
\hline Antinuclear antibody positive (\%) & $214(98.6)$ \\
\hline Anti-dsDNA antibodies positive (\%) & $152(70.0)$ \\
\hline Anti-Smith antibodies positive (\%) & $51(23.5)$ \\
\hline Anti-SSA antibodies positive (\%) & $101(46.5)$ \\
\hline Anti-SSB antibodies positive (\%) & $24(11.1)$ \\
\hline Anti-C1q antibodies positive (\%) & $90(41.5)$ \\
\hline
\end{tabular}

s.d. standard deviation, SLEDAI Systemic Lupus Erythematosus Disease Activity Index, IQR interquartile range, dsDNA double-stranded DNA, SSA Sjogren's syndrome $A$ antigen, SSB Sjogren's syndrome $B$ antigen

manifestations of patients at biopsy. The percentage of glomerular IgM $3+-4+$ in patients with histological Class II, III (including III +V), IV (including IV + V) and $\mathrm{V}$ were $14.3 \%, 13.5 \%, 16.7 \%$ and $8.5 \%$, respectively. Although the percentage of stronger IgM deposition seemed to be lower in Class V, but the difference did not reach statistical significance $(8.5 \%$ in Class V vs. $15.9 \%$ in other Classes; $P=0.201$ ). It was found that the intensity of IgM deposition was correlated with the intensity of IgG and IgA deposition in glomeruli $(r=0.293$, $P<0.001 ; \mathrm{r}=0.456, P<0.001)$. IgM deposition was positively correlated with glomerular $\mathrm{C} 1 \mathrm{q}$ and $\mathrm{C} 3$ deposition moderately $(\mathrm{r}=0.408, P<0.001 ; \mathrm{r}=0.436, P<0.001$, respectively), and inversely correlated with plasma 
Table 2 Renal histopathological profiles of patients with lupus nephritis at renal biopsy

\begin{tabular}{ll}
\hline Characteristics & Value \\
\hline Activity indices score (median, range) & $8(0,19)$ \\
Endocapillary hypercellularity (median, range) & $3(0,3)$ \\
Cellular crescents (median, range) & $0(0,6)$ \\
Karyorrhexis/fibrinoid necrosis (median, range) & $0(0,6)$ \\
Subendothelial hyaline deposits (median, range) & $1(0,3)$ \\
Interstitial inflammatory cell infiltration (median, range) & $1(0,3)$ \\
Glomerular leukocyte infiltration (median, range) & $1(0,12)$ \\
Chronicity indices score (median, range) & $2(0,10)$ \\
Glomerular sclerosis (median, range) & $0(0,3)$ \\
Fibrous crescents (median, range) & $0(0,3)$ \\
Tubular atrophy (median, range) & $1(0,3)$ \\
Interstitial fibrosis (median, range) & $1(0,3)$ \\
\hline
\end{tabular}

Table 3 Renal immunofluoresence profiles of patients with lupus nephritis at renal biopsy

\begin{tabular}{|c|c|}
\hline Characteristics & Value \\
\hline \multicolumn{2}{|c|}{ IgG immunofluorescence intensity, n (\%) } \\
\hline $0+$ & $8(3.7)$ \\
\hline $1+$ & $45(20.7)$ \\
\hline $2+$ & $78(35.9)$ \\
\hline $3+$ & $78(35.9)$ \\
\hline $4+$ & $8(3.7)$ \\
\hline \multicolumn{2}{|c|}{ IgA immunofluorescence intensity, n (\%) } \\
\hline $0+$ & $24(11.1)$ \\
\hline $1+$ & $57(26.3)$ \\
\hline $2+$ & $88(40.6)$ \\
\hline $3+$ & $46(21.2)$ \\
\hline $4+$ & $2(0.9)$ \\
\hline \multicolumn{2}{|c|}{ IgM immunofluorescence intensity, n (\%) } \\
\hline $0+$ & $33(15.2)$ \\
\hline $1+$ & $72(33.2)$ \\
\hline $2+$ & $81(37.3)$ \\
\hline $3+$ & $29(13.4)$ \\
\hline $4+$ & $2(0.9)$ \\
\hline \multicolumn{2}{|c|}{ C3 immunofluorescence intensity, n (\%) } \\
\hline $0+$ & $4(1.8)$ \\
\hline $1+$ & $21(9.7)$ \\
\hline $2+$ & $75(34.6)$ \\
\hline $3+$ & $107(49.3)$ \\
\hline $4+$ & $10(4.6)$ \\
\hline \multicolumn{2}{|c|}{ C1q immunofluorescence intensity, n (\%) } \\
\hline $0+$ & $15(6.5)$ \\
\hline $1+$ & $52(24.0)$ \\
\hline $2+$ & $83(38.2)$ \\
\hline $3+$ & $66(30.4)$ \\
\hline $4+$ & $2(0.9)$ \\
\hline
\end{tabular}

levels of $\mathrm{C} 3$ and $\mathrm{CFH}$ mildly $(\mathrm{r}=-0.138, P=0.043$; $\mathrm{r}=-0.147, P=0.037$, respectively), but no correlation with plasma $\mathrm{C} 1 \mathrm{q}$ levels $(\mathrm{r}=-0.109, P=0.115)$. Among the pathologic indices, glomerular IgM deposition was positively correlated with subendothelial hyaline deposits mildly $(\mathrm{r}=0.136, P=0.045)$ and inversely correlated with interstitial inflammatory cell infiltration $(r=-0.153$, $P=0.025)$. There was no correlation between IgM deposition and proteinuria levels, serum creatine levels or SLEDAI scores (detailed in Table 4).

Glomerular IgM deposition independently contributes to glomerular C3 deposition in patients with $\mathrm{LN}$

Complement activation was one of the mechanisms contributing to renal injury initiated by immune complexes deposition. Since glomerular IgM deposition was not only positively correlated with C3 deposition,

Table 4 Correlations between clinicopathological data and glomerular lgM deposition in lupus nephritis

\begin{tabular}{|c|c|c|}
\hline Parameters & $r$ & $P$ value \\
\hline \multicolumn{3}{|l|}{ Clinical parameters } \\
\hline Age (years) & -0.154 & 0.024 \\
\hline SLEDAI & 0.013 & 0.847 \\
\hline Hemoglobin (g/L) & 0.008 & 0.907 \\
\hline 24-h urine protein (g/24 h) & 0.050 & 0.466 \\
\hline Serum creatinine $(\mu \mathrm{mol} / \mathrm{L})$ & -0.039 & 0.572 \\
\hline Plasma C1q levels (mg/L) & -0.109 & 0.115 \\
\hline Plasma C3 levels (g/L) & -0.138 & 0.043 \\
\hline Plasma CFH levels ( $\mu \mathrm{g} / \mathrm{mL})$ & -0.147 & 0.037 \\
\hline Plasma soluble C5b-9 levels (ng/mL) & 0.009 & 0.899 \\
\hline \multicolumn{3}{|l|}{ Pathological parameters } \\
\hline Histological classes & -0.010 & 0.882 \\
\hline $\mathrm{Al}$ & 0.003 & 0.965 \\
\hline Endocapillary hypercellularity & 0.050 & 0.461 \\
\hline Cellular crescents & -0.064 & 0.345 \\
\hline Karyorrhexis/fibrinoid necrosis & 0.024 & 0.720 \\
\hline Subendothelial hyaline deposits & 0.136 & 0.045 \\
\hline Interstitial inflammatory cell infiltration & -0.153 & 0.025 \\
\hline Glomerular leukocyte infiltration & -0.005 & 0.943 \\
\hline $\mathrm{Cl}$ & -0.022 & 0.745 \\
\hline Glomerular sclerosis & -0.014 & 0.843 \\
\hline Fibrous crescents & -0.012 & 0.860 \\
\hline Tubular atrophy & -0.033 & 0.626 \\
\hline Interstitial fibrosis & -0.062 & 0.360 \\
\hline Glomerular lgG deposition & 0.293 & $<0.001$ \\
\hline Glomerular IgA deposition & 0.456 & $<0.001$ \\
\hline Glomerular C3 deposition & 0.436 & $<0.001$ \\
\hline Glomerular C1q deposition & 0.408 & $<0.001$ \\
\hline
\end{tabular}

CFH complement factor H, SLEDAI Systemic Lupus Erythematosus Disease Activity Index, $\mathrm{Al}$ activity indices, $\mathrm{Cl}$ chronicity indices 
but also correlated with glomerular IgG and IgA deposition, multivariate analysis was used to determine whether IgM contributed to complement activation independently.

As shown in Table 3, in our cohort, almost all the patients had C3 deposition in glomeruli. Among them, $46.1 \%$ patients had relatively mild C3 deposition $(0+-2+)$, and $53.9 \%$ patients had relatively strong C3 deposition $(3+-4+)$. Then multivariate analysis of predictors for strong granular C3 staining was performed using the binary logistic regression model. We found that glomerular IgM deposition independently contributed to glomerular $\mathrm{C} 3$ deposition in patients with $\mathrm{LN}(\mathrm{OR}=2.002$, 95\% CI $1.295-3.094, P=0.002)$ (shown in Table 5).

\section{Association of glomerular IgM deposition and circulating CFH levels in patients with LN}

In animal models, $\mathrm{CFH}^{-1-}$ leads to glomerular IgM deposition [5]. Interestingly, in our cohort, we also found negative correlation between the intensity of glomerular IgM deposition and plasma CFH levels, although the correlation coefficient was low. We further compared circulating CFH levels in patients with different glomerular IgM deposition intensity. Plasma $\mathrm{CFH}$ levels were significantly lower in patients with IgM $3+-4+$ compared with those in patients with IgM $0+-2+(300.4 \pm 155.8 \mu \mathrm{g} / \mathrm{mL}$ vs. $429.9 \pm 187.5$ $\mu \mathrm{g} / \mathrm{mL}, P<0.001)$. While referring to IgG, it was found that plasma $\mathrm{CFH}$ levels were also associated with the intensity of glomerular IgG deposition, but with an opposite tendency compared with the deposition of IgM (Fig. 1).

\section{Association of clinicopathological parameters and the levels of plasma $\mathrm{CFH}$ and glomerular IgM deposition}

According to our previous data, the median plasma $\mathrm{CFH}$ level in healthy controls was $542.8 \mu \mathrm{g} / \mathrm{mL}$ [14]. Then we divided our LN patients into 2 groups: Lower CFH level group (plasma CFH levels $<542.8 \mu \mathrm{g} / \mathrm{mL}$ ) and higher CFH level group (plasma CFH levels $\geq 542.8 \mu \mathrm{g} / \mathrm{mL}$ ). In this cohort, 165 patients had lower plasma CFH levels, and 52 patients had higher plasma CFH levels. In the higher CFH level group, 51 patients had relatively weaker glomerular IgM deposition $(0-2+)$, and only 1 patient had glomerular IgM $3+$.

In the lower $\mathrm{CFH}$ level group, 30 patients had glomerular IgM 3+-4+, while 135 patients had glomerular IgM $0-2+$. We found that patients with stronger glomerular IgM deposition had significantly heavier proteinuria, higher serum creatinine and lower plasma C3 levels $(5.7 \pm 3.1 \mathrm{~g} / \mathrm{d}$ vs. $4.6 \pm 3.3 \mathrm{~g} / \mathrm{d}, P=0.026$; $150.1 \pm 121.0 \mu \mathrm{mol} / \mathrm{L}$ vs. $124.7 \pm 140.6 \mu \mathrm{mol} / \mathrm{L}, P=0.015$; $0.30 \pm 0.2 \mu \mathrm{g} / \mathrm{L}$ vs. $0.44 \pm 0.22 \mu \mathrm{g} / \mathrm{L}, P=0.023$, respectively). There was no significant difference in other indices (detailed in Table 6).

\section{Association of glomerular IgM deposition and renal outcome in patients with LN}

In our cohort, patients with $\mathrm{LN}$ were followed up for a mean duration of $57.3 \pm 58.1$ months after renal biopsy, and the median time between presentation of LN and renal biopsy were 23 months (0-214 months). The median duration of follow-up was 49 months, ranged from 6 to 240 months. We evaluated the association between glomerular IgM deposition and renal survival by Kaplan-Meier survival analysis. It was found that

Table 5 Risk factors for glomerular C3 deposition in patients with lupus nephritis

\begin{tabular}{|c|c|c|c|c|}
\hline \multirow[t]{2}{*}{ Parameter } & \multicolumn{2}{|l|}{ Univariate analysis } & \multicolumn{2}{|c|}{ Multivariate analysis } \\
\hline & OR $(95 \% \mathrm{Cl})$ & $P$ value & OR $(95 \% \mathrm{Cl})$ & $P$ value \\
\hline Sex, men & $0.557(0.262-1.187)$ & 0.130 & - & - \\
\hline Age, per $10 \mathrm{yr}$ & $0.870(0.682-1.108)$ & 0.259 & - & - \\
\hline Urinary protein (g/24 h) & $1.050(0.972-1.135)$ & 0.216 & - & - \\
\hline Serum creatinine $(\mu \mathrm{mol} / \mathrm{L})$ & $1.002(0.999-1.004)$ & 0.171 & - & - \\
\hline Plasma C1q levels ( $\mu \mathrm{g} / \mathrm{mL})$ & $0.993(0.980-1.007)$ & 0.328 & - & - \\
\hline Plasma CFH levels ( $\mu \mathrm{g} / \mathrm{mL})$ & $0.999(0.997-1.000)$ & 0.123 & - & - \\
\hline Plasma C3 levels (g/L) & $0.163(0.047-0.559)$ & 0.004 & $0.243(0.052-1.138)$ & 0.072 \\
\hline Glomerular C1q deposition & $2.890(2.890-4.158)$ & $<0.001$ & $1.940(1.281-2.937)$ & 0.002 \\
\hline Glomerular lgG deposition & $2.238(1.600-3.130)$ & $<0.001$ & 1.908 (1.278-2.848) & 0.002 \\
\hline Glomerular IgA deposition & $2.339(1.677-3.263)$ & $<0.001$ & $1.360(0.915-2.021)$ & 0.129 \\
\hline Glomerular IgM deposition & $2.993(2.073-4.322)$ & $<0.001$ & $2.002(1.295-3.094)$ & 0.002 \\
\hline
\end{tabular}




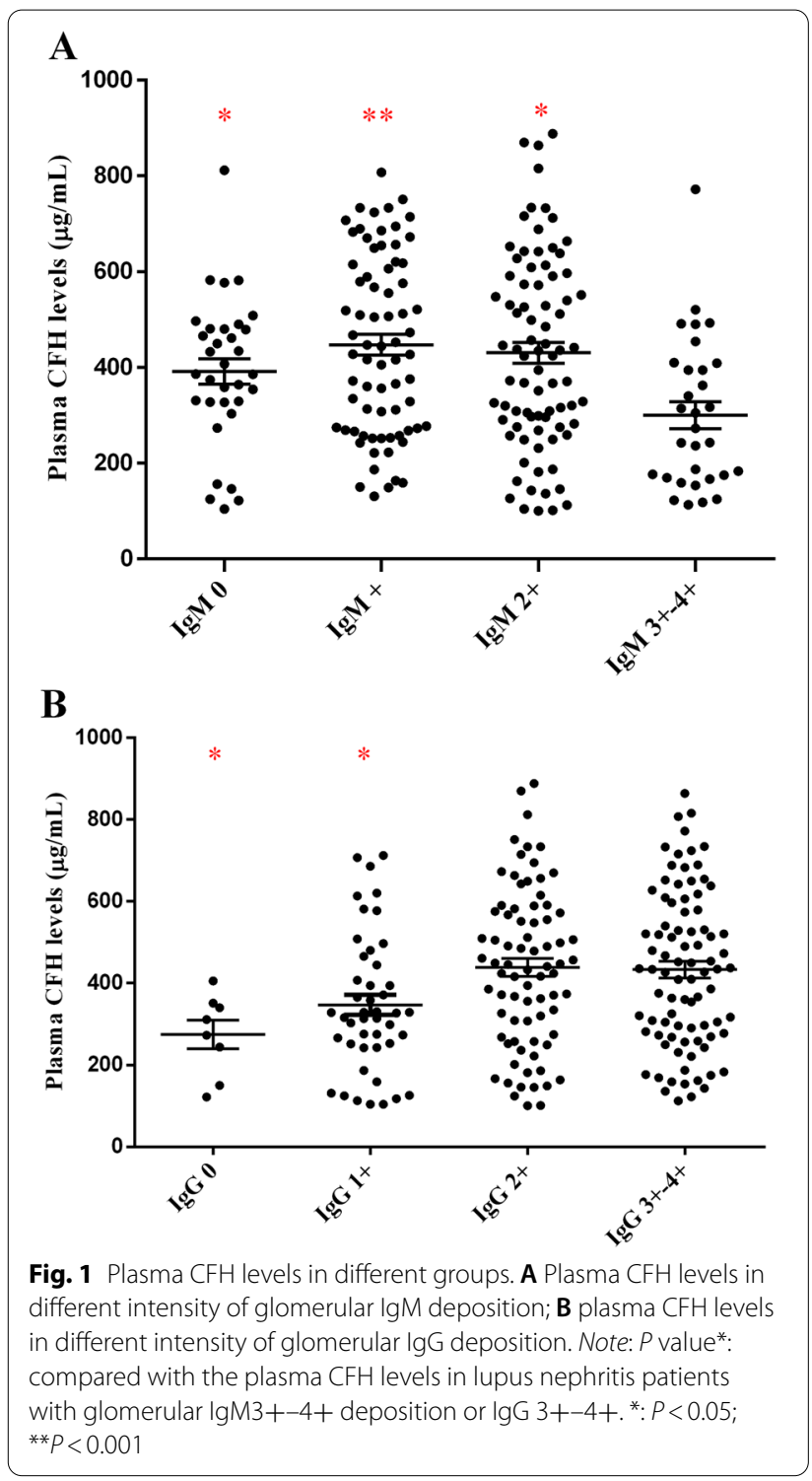

glomerular IgM deposition was not a risk factor for renal outcome in our patients with $\mathrm{LN}(P=0.581, \mathrm{HR}=0.854$, 95\% CI 0.488-1.495). Patients were classified into two groups according to the intensity of glomerular IgM deposition: IgM $0+-2+$ and $\operatorname{IgM} 3+-4+$. However, there was still no significant difference in renal survival between the two groups $(P=0.33)$ (Fig. 2).

\section{Discussion}

Glomerular IgM staining is commonly observed in the mesangial area and on the capillary wall of glomeruli in patients with LN, but its clinical significance has not been elucidated yet. In this large cohort of patients with LN, we firstly reported that glomerular IgM independently contributed to glomerular C3 deposition, and glomerular
IgM deposition intensity was associated with plasma CFH levels.

The deposition of immune complexes and the subsequent complement activation are considered major mechanisms by which tissue injury occurs in LN [15-17]. Many studies have reported the pathogenic role of IgG type autoantibodies, such as anti-dsDNA antibodies, anti-C1q antibodies, anti-mCRP antibodies, in complement activation and renal injuries in LN [18-21]. Natural IgM antibody is generally regarded as an activator of complement classical pathway. Complement activation by IgM antibodies was essential for C3 deposition on apoptotic cells and their uptake by macrophages [22], indicating the involvement of IgM in the pathogenesis of SLE. In this study, we found that the intensity of glomerular deposited IgM was positively correlated with the intensity of glomerular deposited IgG and IgA, and moderately correlated with glomerular deposited C1q and C3. In the further multivariate analysis, glomerular deposited IgG and C1q contributed to glomerular C3 deposition, which supported that nephritogenic IgG autoantibodies mediated complement classical pathway was activated in renal tissue in LN. What's more, IgM rather than IgA contributed to glomerular C3 deposition independently. Our findings firstly indicated that the abundant deposited IgM in glomeruli in LN was involved in the complement activation and pathogenicity.

Deletion of $\mathrm{CFH}$ results in uncontrolled complement alternative pathway activation and glomerular injury. $\mathrm{CFH}^{-1-}$ mice is an animal model of non-sclerotic and nonimmune-complex glomerular disease. CFH deficiency induced IgM deposition on endothelial cells and subendothelial areas. Panzer et al. crossed $\mathrm{CFH}^{-1-}$ mice with $\mu M T$ mice (mice unable to produce IgM) and demonstrated that IgM contributed to the progression of glomerular damage induced by $\mathrm{CFH}$ deficiency [5]. In active LN, plasma CFH levels decreased to lower levels [14]. Interestingly, in this study we found that glomerular IgM deposition was inversely correlated with plasma levels of $\mathrm{CFH}$, plasma $\mathrm{CFH}$ levels were significantly lower in patients with stronger IgM deposition $(3+-4+)$ compared with those in patients with weaker IgM deposition $(0+-2+)$, and stronger glomerular IgM deposition was more often observed in lower $\mathrm{CFH}$ group. Our results proposed that in patients with $\mathrm{LN}, \mathrm{CFH}$ deficiency seemed to induce extra glomerular IgM deposition too. IgM within the glomerulus was considered as a downstream event occurring secondary to glomerular damage. Some clinical studies of patients with nephrotic syndrome demonstrated more severe manifestations in association with secondary glomerular IgM deposition [23-26]. However, it is clear that not all patients with glomerular disease develop IgM deposition. According 
Table 6 Comparisons of clinical, laboratory and pathological data in patients with lower plasma CFH level group

\begin{tabular}{|c|c|c|c|}
\hline & \multicolumn{3}{|c|}{ Lower plasma CFH level group } \\
\hline & $\begin{array}{l}\lg M 3+-4+ \\
N=30\end{array}$ & $\begin{array}{l}\operatorname{lgM} 0-2+ \\
\mathrm{N}=135\end{array}$ & $P$ \\
\hline \multicolumn{4}{|l|}{ Clinical evaluation } \\
\hline Gender (male \%) & $6(20.0 \%)$ & $17(12.6 \%)$ & 0.380 \\
\hline Age (mean \pm s.d.) (years) & $31.1 \pm 10.5$ & $33.3 \pm 11.8$ & 0.411 \\
\hline SLEDAI (mean \pm s.d.) & $17.2 \pm 5.2$ & $18.0 \pm 5.8$ & 0.408 \\
\hline \multicolumn{4}{|l|}{ Laboratory assessment } \\
\hline Hemoglobin (mean \pm s.d.) $(\mathrm{g} / \mathrm{L})$ & $100.9 \pm 26.5$ & $99.6 \pm 25.7$ & 0.859 \\
\hline Urine protein (median; IQR) (g/24 h) & $5.7 \pm 3.1$ & $4.6 \pm 3.3$ & 0.026 \\
\hline $\operatorname{Scr}($ mean \pm s.d. $)(\mu \mathrm{mol} / \mathrm{L})$ & $150.1 \pm 121.0$ & $124.7 \pm 140.6$ & 0.015 \\
\hline Plasma C1q (mean \pm s.d.) $(\mu \mathrm{g} / \mathrm{mL})$ & $33.2 \pm 18.8$ & $33.2 \pm 18.8$ & 0.191 \\
\hline Plasma C3 levels (mean \pm s.d.) (g/L) & $0.30 \pm 0.2$ & $0.44 \pm 0.22$ & 0.023 \\
\hline Plasma C4 levels (mean \pm s.d.) (g/L) & $0.23 \pm 0.17$ & $0.21 \pm 0.18$ & 0.756 \\
\hline Plasma CFH levels (mean \pm s.d.) $(\mu \mathrm{g} / \mathrm{mL})$ & $294.6 \pm 131.1$ & $338.7 \pm 124.3$ & 0.052 \\
\hline Plasma soluble C5b-9 levels (mean \pm s.d.) $(\mathrm{ng} / \mathrm{mL})$ & $1225.9 \pm 571.2$ & $1271.4 \pm 657.8$ & 0.785 \\
\hline \multicolumn{4}{|l|}{ Pathological parameters } \\
\hline Al (median; IQR) & $8(6,10)$ & $8(4,11)$ & 0.695 \\
\hline Endocapillary hypercellularity & $3(2,3)$ & $3(1,3)$ & 0.620 \\
\hline Cellular crescents & $0(0,2)$ & $0(0,2)$ & 0.591 \\
\hline Karyorrhexis/fibrinoid necrosis & $0(0,2)$ & $0(0,2)$ & 0.640 \\
\hline Subendothelial hyaline deposits & $1(1,3)$ & $1(0,2)$ & 0.118 \\
\hline Interstitial inflammatory cell infiltration & $1(1,1)$ & $1(1,2)$ & 0.856 \\
\hline Glomerular leukocyte infiltration & $1(1,1)$ & $1(0,1)$ & 0.454 \\
\hline $\mathrm{Cl}$ (median; IQR) & $2.5(2,4)$ & $2(2,4)$ & 0.368 \\
\hline Glomerular sclerosis & $0(0,1)$ & $0(0,1)$ & 0.970 \\
\hline Fibrous crescents & $0(0,1)$ & $0(0,0)$ & 0.228 \\
\hline Tubular atrophy & $1(1,1)$ & $1(1,1)$ & 0.481 \\
\hline Interstitial fibrosis & $1(1,1)$ & $1(1,1)$ & 0.604 \\
\hline
\end{tabular}

CFH complement factor $\mathrm{H}$, s.d. standard deviation, SLEDAI Systemic Lupus Erythematosus Disease Activity Index, IQR interquartile range, $\mathrm{Al}$ activity indices, $\mathrm{Cl}$ chronicity indices

to the result of animal model and this clinical study, the correlationship of secondary IgM deposition and plasma CFH levels was deserved to be explored in other glomerulonephritis.

LN is characterized by the "full-house" pattern of immune deposits, IgM deposition in LN was obviously not only induced secondarily. In our cohort, it was not found obvious correlations between IgM deposition and clinicopathological parameters. As discussed above, lower CFH levels might cause extra IgM deposition. We further compared clinicopathological parameters in patients with lower CFH levels, and found that patients with stronger glomerular IgM deposition had significantly heavier proteinuria, higher serum creatinine and lower plasma C3 levels in the analysis subgroup. Our results indicated that secondarily deposited IgM in LN might also demonstrate more severe manifestations.

On the basis of the above relevance of glomerular IgM deposition in patients with $\mathrm{LN}$, it is important to evaluate the predictive value of glomerular IgM deposition for long-term renal outcome in LN. Recently, several studies showed that IgM deposition predicted renal outcome in patients with IgAN, FSGS and diabetic nephropathy [7, $9,27,28]$. However, owing to the relatively good treatment response of $\mathrm{LN}$ and our relatively small follow-up population, we failed to draw any convincing conclusions regarding the predictive value of glomerular IgM deposition in our study.

Our study had several limitations. First, it was a retrospectively observational study, a cause-effect relationship could not be established. Second, this is the first report revealing clinical significance of IgM deposition in patients with $\mathrm{LN}$, findings from this single-center study require validation from multicenter studies with larger cohorts. Third, a "full-house" pattern of immune deposits in LN caused complex pathophysiological process in complement activation, IgM deposition involved in kidney injury progression was not fully interpreted. 


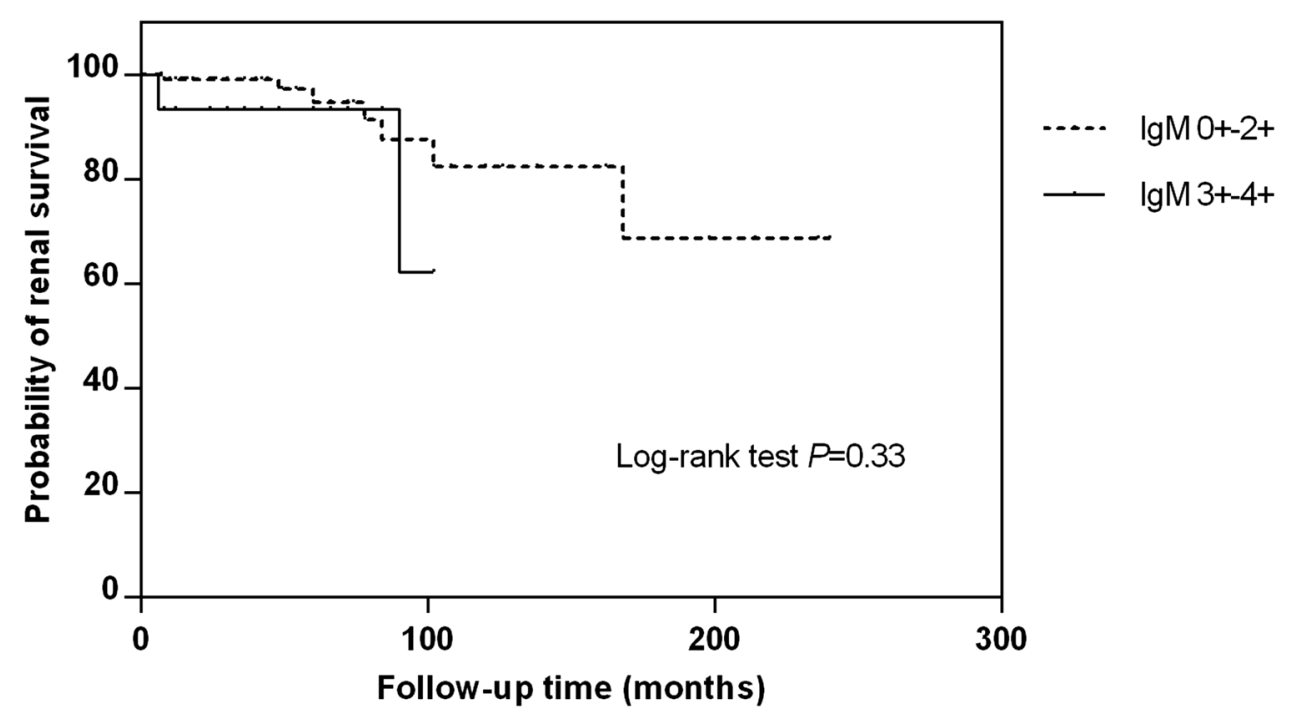

Patients at risk

$\lg \mathrm{M} 0+-2+186$

$\lg \mathrm{M} 3+-4+31$

33

3

$\begin{array}{ll}9 & 0 \\ 0 & 0\end{array}$

Fig. 2 The correlation between intensity of glomerular lgM deposition and renal survival in patients with lupus nephritis

In conclusion, this study shows that glomerular IgM independently contributed to glomerular C3 deposition, and glomerular IgM deposition intensity was associated with plasma CFH levels. The findings indicate the involvement of glomerular IgM in complement activation and renal injury.

\section{Methods}

Informed consent was obtained for blood sampling and renal biopsy from each patient. For participants under 16 years old, written informed consent was provided by a parent or guardian. In addition, all clinical test results were also obtained after patients gave their consent to use them for research purposes. The research was in compliance with the Declaration of Helsinki and approved by IEC for Clinical Research of Southeast University affiliated Zhongda Hospital (No. 2013-075).

\section{Patients}

A total of 217 consecutive patients with renal biopsyproven lupus nephritis diagnosed at Zhongda Hospital affiliated to Southeast University from 6 September 2013 to 2 March 2019 were enrolled in this study. The patients all fulfilled the 1997 ACR revised criteria for SLE [29].

\section{Clinical evaluations}

The following clinical data were collected and analysed: gender, age, fever, anaemia, leucocytopenia, thrombocytopenia, haematuria, leukocyturia, 24-h proteinuria, serum creatinine and hemoglobulin. Clinical disease activity was assessed using the SLEDAI [30]. eGFR was calculated using a Scr-based equation adjusted for coefficients for age and gender by modified abbreviated MDRD equation based on data from Chinese CKD patients: eGFR ( $\mathrm{mL} / \mathrm{min}$ per $\left.1.73 \mathrm{~m}^{2}\right)=175 \times[\mathrm{Scr} \quad(\mathrm{mg} / \mathrm{dL})]^{-1.234} \times \operatorname{age}^{-0.179} \times(0.79$ if female) [31].

The criteria for clinical remission were the same as we reported previously [14]. The remission of lupus nephritis includes complete remission and partial remission. Complete remission was defined as a urinary protein excretion of $<0.3 \mathrm{~g} /$ day, with normal urinary sediments (red blood cell $<3 /$ high power $(\mathrm{HP})$, white blood cell $<5 / H P$ ), serum albumin and renal function. Partial remission was defined as the presence of any one of the following features: a decrease in serum creatinine to below $130 \mu \mathrm{mol} / \mathrm{L}$ for patients with a baseline serum creatinine of $\geq 130 \mu \mathrm{mol} / \mathrm{L}$, but $\leq 260 \mu \mathrm{mol} / \mathrm{L}$; a decrease in serum creatinine by $>50 \%$ for patients with a baseline serum creatinine of $>260 \mu \mathrm{mol} / \mathrm{L}$; a decrease in urinary protein excretion by $>50 \%$, and below $3.0 \mathrm{~g} / 24 \mathrm{~h}$, with a serum albumin of $\geq 30 \mathrm{~g} / \mathrm{L}$ and stable renal function.

The patients were followed up in our outpatient clinic specified for LN. The renal endpoints were defined as end-stage renal disease (ESRD) or doubling of serum creatinine. 


\section{Blood samples}

Plasma and sera from patients with LN were obtained from peripheral blood at the time of renal biopsy. All the blood samples were stored at $-80^{\circ} \mathrm{C}$ until use.

\section{Laboratory assessment}

Serum ANAs, anti-dsDNA antibodies, anti-extractable nuclear antigen antibodies, including anti-Sm, anti-SSA, anti-SSB antibodies, were detected using immunodotting assays (EUROIMMUN, Lübeck, Germany; normal range). Plasma $\mathrm{C} 3$ was determined using a rate nephelometry assay (BeckmanCoulter, IMMAGE, USA; normal range $>0.85 \mathrm{~g} / \mathrm{L})$.

Anti-C1q IgG autoantibodies were detected using a previously published ELISA method [32]. The results were recorded as the net optical absorbance (average value of antigen wells minus average value of antigenfree wells) at $490 \mathrm{~nm}$ in an ELISA reader (BioRad 550, Japan) and expressed as percentage of the known positive sample. The cutoff value was set as the mean +2 SD of healthy blood donors.

\section{Quantification of plasma levels of complement components}

Plasma concentrations of human complement components were determined by enzyme-linked immunosorbent assay, including plasma $\mathrm{C} 1 \mathrm{q}$, plasma $\mathrm{CFH}$ and soluble C5b-9 (Quidel Corporation, San Diego, CA). The detection of plasma soluble C5b-9 was assayed in accordance with the manufacturer's instructions.

The method to detect plasma C1q was modified as previously described [32]. Rabbit anti-human C1q polyclonal antibodies (Dako, Denmark) were coated on to the microtiter plates (Nunc Immunoplate, Roskilde, Denmark) overnight at $4{ }^{\circ} \mathrm{C}$. Then the wells were washed 3 times with $0.01 \mathrm{M}$ phosphate-buffered saline (PBS) containing $0.1 \%$ Tween 20 (PBST) and blocked with PBSTcontaining $1 \%$ bovine serum albumin (BSA). After standards and serum samples added, horseradish peroxidase-conjugated goat anti-human $\mathrm{Clq}$ monoclonal antibody (Abcam, US) was added and incubated. The reaction was developed with tetramethylbenzi-dine (TMB) liquid substrate system and was stopped with $1 \mathrm{M} \mathrm{H}_{2} \mathrm{SO}_{4}$. The results were recorded as the net optical absorbance at 450 and $570 \mathrm{~nm}$ in an ELISA reader (Bio-Rad550, Japan).

The method of detecting plasma $\mathrm{CFH}$ was the same as previously described [14]. The CFH level of each sample was calculated using Curve Expert 1.3 (Hyams Development, http://www.curveexpert.net/). All assays were run in duplicate, and when standard errors were $>10 \%$, samples were routinely re-analyzed. Serial concentrations of commercially available highly purified human factor $\mathrm{H}$ were used to develop a standard curve. The linear portion of the curve was subsequently used for the measurement of plasma factor $\mathrm{H}$.

\section{Renal histopathology}

The renal biopsy specimens were examined by light microscopy, direct immunofluorescence and electron microscopy. LN was re-classified according to the International Society of Nephrology/Renal Pathology Society (ISN/RPS) 2003 classification system [33]. All renal histopathological data of 217 patients with renal biopsy-proven lupus nephritis, were reviewed by two pathologists with 20-year experience. The pathologists classified and scored the biopsies separately, blinded to patients' data and the scores of the other observer. Differences in scoring between the pathologists were resolved by rereviewing the biopsies and reaching a consensus.

\section{Intra- and inter-reader reliability}

The two pathologists who undertook the analyses of the pathological data, were unaware of the patients' details. Each evaluation was performed in triplicate and the mean of the values were reported. In addition, in each patient's pathological data evaluation, two expert pathologists reported their results. The inter-rater reliability for immunofluorescence IgG and IgM scores was good, Cohen's kappa $=0.953(P<0.001)$, Cohen's kappa $=0.951$ $(P<0.001)$, respectively. The intra-rater reliability for immunofluorescence IgG and IgM scores from one pathologist was excellent, ICC $=0.962(95 \%$ CI $0.952-$ 0.971 ), ICC $=0.980$ (95\% CI $0.974-0.985$ ), respectively. And the intra-rater reliability for immunofluorescence IgG and IgM scores from the other pathologist was also good, ICC $=0.946$ (95\% CI 0.931-0.958), ICC $=0.965$ (95\% CI 0.955-0.973), respectively.

\section{Light microscopy examination}

Renal biopsy specimens were fixed in $4.5 \%$ buffered formaldehyde for light microscopy. Consecutive serial $3 \mu \mathrm{m}$ sections were used for histological staining. Stains employed included hematoxylins and eosin (H\&E), periodic acid-Schiff, silver methenamine (Meth) and Masson's trichrome.

\section{Immunofluorescence examination}

The intensity of immunofluorescence for IgG, IgA, IgM, C3, C1q, fibrin, kappa and lambda deposits were semiquantitatively graded from 0 to 4 , respectively. Staining intensity was expressed as follows: 0 , invisible; $1+$, ambiguous under a low-power lens and apparent under a high-power lens; $2+$, apparent under a low-power lens and clear under a high-power lens; $3+$, clear under a lowpower lens and bright under a high-power lens; and 4+, dazzling under a high-power lens. 


\section{Electron microscopy}

Renal biopsy specimens were fixed in $2.5 \%$ paraformaldehyde for electron microscopy. After embedded in epon, ultrathin sections were mounted on metal grids and stained with uranyl acetate before viewed in a transmission electron microscope (JEM-1230; JEOL,Tokyo, Japan).

\section{Statistical analysis}

Statistical software SPSS 25.0 (SPSS, Chicago, IL, USA) was employed for all the statistical analysis. Quantitative data were expressed as mean \pm s.d., median with interquartile range (IQR), median with range, or number (\%). One-way analysis of variance was used for the same continuous data in different groups. Differences of quantitative variables between groups were assessed using the $t$ test (for normally distributed data) or Mann-Whitney U test (for non-normally distributed data). Logistic regression analysis was carried out to predict glomerular C3 deposition. Survival analysis was performed using the log-rank test. Statistical significance was considered as $P<0.05$.

\begin{abstract}
Abbreviations
LN: Lupus nephritis; SLE: Systemic lupus erythematosus; CFH: Complement factor H; FSGS: Focal segmental glomerulosclerosis; IgAN: IgA nephropathy; DN: Diabetic nephropathy; SLEDAI: Systemic Lupus Erythematosus Disease Activity Index; dsDNA: Double-stranded DNA; SSA: Sjogren's syndrome A antigen; SSB: Sjogren's syndrome B antigen; Al: Activity indices; Cl: Chronicity indices; PBS: Phosphate-buffered saline; BSA: Bovine serum albumin; ISN/RPS International Society of Nephrology/Renal Pathology Society; IQR: Interquartile range; OR: Odd ratio; $\mathrm{Cl}$ : Confidence interval; Al: Activity indices; $\mathrm{Cl}$ : Chronicity indices.
\end{abstract}

\section{Acknowledgements}

Not applicable.

\section{Authors' contributions}

FMW drafted the manuscript and did the whole revision process. JRY, LZ and YZ participated in collecting the related clinical and pathological data. JZ conducted partly statistical analysis. XWY acted as the corresponding author did the whole study design and the manuscript revision. $B C L$ acted as the co-corresponding author and did the partly study design and the manuscript revision. All authors read and approved the final manuscript.

\section{Funding}

This work was supported by grants from the National Natural Science Foundation of China (Nos. 82060132, 81400704, 81300587), Science and Technology Assistance Project of Science and Technology Department of Xinjiang Autonomous Region (No. 2020E0276) and Nanjing municipal commission of health and family planning (No. 7990000097).

\section{Availability of data and materials}

Further clinical data and images of this case are available from the corresponding author upon reasonable request.

\section{Declarations}

Ethics approval and consent to participate

Informed consent was obtained for blood sampling and renal biopsy from each patient. For participants under 16 years old, written informed consent was provided by a parent or guardian. In addition, all clinical test results were also obtained after patients gave their consent to use them for research purposes. The research was in compliance with the Declaration of Helsinki and approved by the local ethics committees (No. 2013-075).

\section{Consent for publication}

Not applicable.

\section{Competing interests}

The authors declare that they have no competing interests.

\section{Author details}

${ }^{1}$ Institute of Nephrology, Zhong Da Hospital, Southeast University School of Medicine, Nanjing, China. ${ }^{2}$ Department of Nephrology, The Fifth Affiliated Hospital of Xinjiang Medical University, Urumqi 830000, Xinjiang, China. ${ }^{3}$ Department of Public Health, Aarhus University, Aarhus, Denmark. ${ }^{4}$ Department of Nephrology, Provincial Hospital Affiliated to Shandong University, Jinan 250021, Shandong, China. ${ }^{5}$ Department of Nephrology, Provincial Hospital Affiliated to Shandong First Medical University, Jinan 250021, Shandong, China.

Received: 18 February 2021 Accepted: 22 November 2021

Published online: 07 December 2021

\section{References}

1. Almaani S, Meara A, Rovin BH. Update on lupus nephritis. Clin J Am Soc Nephrol. 2017;12:825-35.

2. Yu F, Haas M, Glassock R, Zhao MH. Redefining lupus nephritis: clinical implications of pathophysiologic subtypes. Nat Rev Nephrol. 2017;13:483-95.

3. Lech M, Anders HJ. The pathogenesis of lupus nephritis. J Am Soc Nephrol. 2013;24:1357-66.

4. Wenderfer SE, Eldin KW. Lupus nephritis. Pediatr Clin North Am. 2019;66:87-99.

5. Panzer SE, Laskowski J, Renner B, Kulik L, Ljubanovic D, Huber KM, Zhong W, Pickering MC, Holers VM, Thurman JM. IgM exacerbates glomerular disease progression in complement-induced glomerulopathy. Kidney Int. 2015;88:528-37.

6. Strassheim D, Renner B, Panzer S, Fuquay R, Kulik L, Ljubanovic D, Holers VM, Thurman JM. IgM contributes to glomerular injury in FSGS. J Am Soc Nephrol. 2013;24:393-406.

7. Pacic A, Senjug P, Bacalja J, Tisljar M, Horvatic I, Bulimbasic S, Knotek M, Galesic K, Galesic LD. IgM as a novel predictor of disease progression in secondary focal segmental glomerulosclerosis. Croat Med J. 2017;58:281-91.

8. Zhang YM, Gu QH, Huang J, Qu Z, Wang X, Meng LQ, Wang F, Liu G, Cui Z, Zhao MH. Clinical significance of IgM and C3 glomerular deposition in primary focal segmental glomerulosclerosis. Clin J Am Soc Nephrol. 2016;11:1582-9.

9. Tang X, Li H, Li L, Zhang J, Xu H, Li L, Liu F. The Clinical impact of glomerular immunoglobulin $\mathrm{M}$ deposition in patients with type 2 diabetic nephropathy. Am J Med Sci. 2018;356:365-73.

10. Platt JL, Cascalho M. IgM in the kidney: a multiple personality disorder. Kidney Int. 2015;88:439-41.

11. Hesketh EE, Dransfield I, Kluth DC, Hughes J. Circulating IgM requires plasma membrane disruption to bind apoptotic and non-apoptotic nucleated cells and erythrocytes. PLoS ONE. 2015;10:e0131849.

12. Reversat A, Sixt M. IgM's exit route. J Exp Med. 2018;215:2959-61.

13. Klimovich VB. IgM and its receptors: structural and functional aspects. Biochemistry (Mosc). 2011;76:534-49.

14. Wang FM, Yu F, Tan Y, Song D, Zhao MH. Serum complement factor $\mathrm{H}$ is associated with clinical and pathological activities of patients with lupus nephritis. Rheumatology (Oxford). 2012;51:2269-77. 
15. Waldman M, Madaio MP. Pathogenic autoantibodies in lupus nephritis. Lupus. 2005;14:19-24.

16. Foster MH. T cells and B cells in lupus nephritis. Semin Nephrol. 2007;27:47-58.

17. Yap DY, Yung S, Chan TM. Lupus nephritis: an update on treatments and pathogenesis. Nephrology (Carlton). 2018;23(Suppl 4):80-3.

18. Li QY, Li HY, Fu G, Yu F, Wu Y, Zhao MH. Autoantibodies against C-reactive protein influence complement activation and clinical course in lupus nephritis. J Am Soc Nephrol. 2017:28:3044-54.

19. Yang XW, Tan Y, Yu F, Zhao MH. Combination of anti-C1q and anti-dsDNA antibodies is associated with higher renal disease activity and predicts renal prognosis of patients with lupus nephritis. Nephrol Dial Transplant. 2012:27:3552-9.

20. Linnik MD, Hu JZ, Heilbrunn KR, Strand V, Hurley FL, Joh T. Relationship between anti-double-stranded DNA antibodies and exacerbation of renal disease in patients with systemic lupus erythematosus. Arthritis Rheum. 2005;52:1129-37.

21. Pang $Y$, Yang $X W$, Song $Y, Y u$ F, Zhao MH. Anti-C1q autoantibodies from active lupus nephritis patients could inhibit the clearance of apoptotic cells and complement classical pathway activation mediated by C1q in vitro. Immunobiology. 2014;219:980-9.

22. Quartier P, Potter PK, Ehrenstein MR, Walport MJ, Botto M. Predominant role of IgM-dependent activation of the classical pathway in the clearance of dying cells by murine bone marrow-derived macrophages in vitro. Eur J Immunol. 2005;35:252-60.

23. Swartz SJ, Eldin KW, Hicks MJ, Feig DI. Minimal change disease with IgM+ immunofluorescence: a subtype of nephrotic syndrome. Pediatr Nephrol. 2009;24:1187-92.

24. Myllymaki J, Saha H, Mustonen J, Helin H, Pasternack A. IgM nephropathy: clinical picture and long-term prognosis. Am J Kidney Dis. 2003:41:343-50.

25. Alexopoulos E, Papagianni A, Stangou M, Pantzaki A, Papadimitriou M. Adult-onset idiopathic nephrotic syndrome associated with pure diffuse mesangial hypercellularity. Nephrol Dial Transplant. 2000;15:981-7.
26. Al RD, Al-Hussain TO, Awad HS, Saadeh SA, Al-Hassoun IA, Al-Shareef TA. Clinical significance of IgM deposition in pediatric minimal change disease. Int J Pediatr Adolesc Med. 2019;6:146-50.

27. Mirioglu S, Caliskan Y, Ozluk Y, Dirim AB, Istemihan Z, Akyildiz A, Yazici $H$, Turkmen A, Kilicaslan I, Sever MS. Co-deposition of IgM and C3 may indicate unfavorable renal outcomes in adult patients with primary focal segmental glomerulosclerosis. Kidney Blood Press Res. 2019;44:961-72.

28. Heybeli C, Oktan MA, Yildiz S, Arda HU, Unlu M, Cavdar C, Sifil A, Celik A, Sarioglu S, Camsari T. Clinical significance of mesangial lgM deposition in patients with IgA nephropathy. Clin Exp Nephrol. 2019;23:371-9.

29. Hochberg MC. Updating the American College of Rheumatology revised criteria for the classification of systemic lupus erythematosus. Arthritis Rheum. 1997:40:1725.

30. Bombardier C, Gladman DD, Urowitz MB, Caron D, Chang CH. Derivation of the SLEDAI. A disease activity index for lupus patients. The Committee on Prognosis Studies in SLE. Arthritis Rheum. 1992;35:630-40.

31. Levey AS, Coresh J, Bolton K, Culleton B, Harvey KS, Ikizler TA, Johnson CA, Kausz A, Kimmel PL, Kusek J, Levin A. K/DOQI clinical practice guidelines for chronic kidney disease: evaluation, classification, and stratification. Am J Kidney Dis. 2002:39:S1-266.

32. Tan Y, Song D, Wu LH, Yu F, Zhao MH. Serum levels and renal deposition of C1q complement component and its antibodies reflect disease activity of lupus nephritis. Bmc Nephrol. 2013;14:63.

33. Weening JJ, D'Agati VD, Schwartz MM, Seshan SV, Alpers CE, Appel GB, Balow JE, Bruijn JA, Cook T, Ferrario F, et al. The classification of glomerulonephritis in systemic lupus erythematosus revisited. Kidney Int. 2004:65:521-30.

\section{Publisher's Note}

Springer Nature remains neutral with regard to jurisdictional claims in published maps and institutional affiliations.
Ready to submit your research? Choose BMC and benefit from:

- fast, convenient online submission

- thorough peer review by experienced researchers in your field

- rapid publication on acceptance

- support for research data, including large and complex data types

- gold Open Access which fosters wider collaboration and increased citations

- maximum visibility for your research: over $100 \mathrm{M}$ website views per year

At BMC, research is always in progress.

Learn more biomedcentral.com/submissions 